\title{
Bridging the gap between transcriptome and connectome
}

\author{
Alex Fornito ${ }^{1,2}$, Aurina Arnatkevičiūtè ${ }^{1}$, Ben D. Fulcher ${ }^{1,3}$ \\ ${ }^{1}$ Brain and Mental Health Research Hub, Monash Institute of Cognitive and Clinical Neurosciences, Monash \\ University, Victoria, Australia \\ ${ }^{2}$ Monash Biomedical Imaging, Monash University, Victoria, Australia \\ ${ }^{3}$ School of Physics, University of Sydney, New South Wales, Australia
}

Corresponding author: Alex Fornito, alex.fornito@monash.edu, twitter: @AFornito

\begin{abstract}
The recent construction of brain-wide gene expression atlases, which measure the transcriptional activity of thousands of genes in many different anatomical locations, has made it possible to connect spatial variations in gene expression to distributed properties of connectome structure and function. These analyses have revealed that spatial patterning of gene expression and neuronal connectivity are closely linked, following broad spatial gradients that track regional variations in microcircuitry, inter-regional connectivity and functional specialization. Superimposed on these gradients are more specific associations between gene expression and connectome topology that appear conserved across diverse species and resolution scales. These findings highlight the utility of brain-wide gene expression atlases for bridging the gap between molecular function and large-scale connectome organization in health and disease.
\end{abstract}

Keywords: MRI; DNA; complex network; microarray; graph analysis; hub 


\section{Introduction}

The full set of elements and connections comprising a nervous system is called a connectome [1]. A connectome can be represented as a network or graph, in which each neural element is a node and each connection between pairs of elements is an edge (Box 1). Network analysis has been used extensively to characterise the properties of connectomes constructed in species ranging from the nematode worm Caenorhabditis elegans [2] to human [3], and measured at scales ranging from individual neurons and synapses to white matter bundles linking macroscopic brain regions [4,5]. This work has shown that connectomes have a complex network topology with many properties that are conserved across species and scales (Box 1) [6].

This striking conservation of connectome topology has been attributed to common selection pressures, such as the competitive drive to minimize network wiring costs while promoting complex, adaptive function [7]. Physical and geometric constraints on the spatial embedding of nervous systems can explain some, but not all, aspects of connectome topology [8-11], suggesting an additional role for genetic influences. Accordingly, human twin studies have shown that topological properties of brain networks, including those quantifying specific aspects of network economy, are heritable [12-15].

How can we identify the genes that contribute to variations in connectome structure and function? In humans, the most common method has involved testing for associations between inter-individual variations in a given brain network phenotype and structural variation of DNA, either through candidate variant studies, which require strong a priori hypotheses concerning the genes involved, or through genome-wide association, which requires very large samples to achieve sufficient statistical power. A wider range of approaches is available in small model species (e.g., $[16,17])$, but these are often difficult to scale to higher mammals.

Here, we consider the merits of a new approach for linking gene function to neural phenotypes measured across the entire brain. This approach leverages brain-wide atlases of gene expression, which quantify the transcriptional activity of thousands of genes across many different anatomical locations [18-20] (Box 2). The broad anatomical and genomic coverage of these atlases makes it possible to comprehensively map the molecular correlates of spatially distributed network properties, thus helping to bridge the gap between transcriptome and connectome.

We begin by outlining basic concepts related to how gene expression is measured and analyzed (see also Boxes 2,3). We then review evidence indicating that the brain's transcriptional architecture is dominated by broad spatial gradients that are tied to variations in cellular and circuit architecture, upon which are superimposed more specific transcriptional signatures of network connectivity and topology. Collectively, this work points to a close link between the spatial patterning of gene expression, neuronal microcircuitry, interregional connectivity, and functional specialization of the brain, and offers new insights into both normal brain organization and disease (Box 4). 


\section{Mapping the brain's transcriptional landscape}

Variations in the nucleotide sequences that constitute an individual's DNA influence gene expression and protein abundance, thereby affecting cellular function and ultimately contributing to phenotypic variability across a population. The synthesis of proteins from DNA is a complex multi-stage process [21]. The two most relevant stages for present purposes are transcription and translation. Transcription is the first stage of gene expression and begins when RNA polymerase reads an unwound segment of DNA to create a complimentary RNA strand called a transcript which, in the case of a protein-coding gene, is messenger RNA (mRNA). Translation begins when mRNA leaves the cell nucleus and reaches the ribosome core, where the transcript is decoded to synthesise amino acid chains that ultimately fold into proteins.

Gene expression is commonly measured indirectly from mRNA levels, which index a gene's transcriptional activity. Ultimately however, it is the translational activity of a cell that determines protein abundance. Transcriptional and translational activity can decouple, and the relationship between gene transcription and protein abundance is not always straightforward [21,22]. The advantage of measuring gene transcription is that it is relatively easier to quantify using high-throughput assays. Existing transcriptional atlases vary in terms of the expression assays used, their tissue specificity, and their anatomical coverage (reviewed in [20]) (Box 2).

Following appropriate processing of the gene expression atlas data [23], transcriptional patterns can be characterized at several levels, including individual brain regions, pairs of brain regions (correlated gene expression, CGE), or pairs of genes (gene coexpression) (Box 2). Spatial or topological variations in the expression measures derived from these approaches can then be correlated with spatial or topological variations in some property of brain structure or function, such as regional volume or inter-regional connectivity (Box 2). As such, these analyses characterize how the spatial patterning of gene expression tracks spatial variations in a given neural phenotype (Boxes 2,3 ). This goal differs from the traditional focus of behavioural genetic and genome-wide association studies, which is to understand how genes contribute to phenotypic variability across individuals.

Atlas-based analyses typically involve testing for spatial correlations between a given connectome property and gene expression across hundreds or thousands of genes. For example, the Allen Mouse Brain Atlas (AMBA) of gene expression comprises measures for over 19,000 genes quantified with cellular resolution in each tissue slice acquired every $200 \mu \mathrm{m}$ through a full brain volume [18,24], and the Allen Human Brain Atlas (AHBA) comprises measures for over 20,000 genes quantified across 3702 different anatomical locations and six different brains [19]. Each gene in these atlases can be scored according to its spatial correlation with the connectome property of interest. Since many genes are functionally related and have coupled expression patterns, the multiple comparison burden can be reduced by conducting inference at the level of functional classes of genes, rather than the individual genes themselves, using statistical tests of enrichment (e.g., 
evaluating whether the mean gene score in a functional class is higher than expected by chance). One popular hierarchy of gene-to-function annotations is the gene ontology (GO) [25]; the Kyoto Encyclopedia of Genes and Genomes (KEGG) is another [26] (for caveats to this approach, see [27-29]).

\section{Spatial gradients dominate the brain's transcriptional and connectional architecture}

One of the most striking patterns observed in brain-wide expression atlases is a strong spatial autocorrelation, such that regions in close physical proximity have more similar transcriptional profiles. This effect has been reported in the human cortex [19,23,30], mouse brain [31,32] and head of $C$. elegans [33], and parallels a similar distance-dependence of connectivity, such that nearby neural elements have a higher probability of connection [9,31,33] (Figure 1a-d).
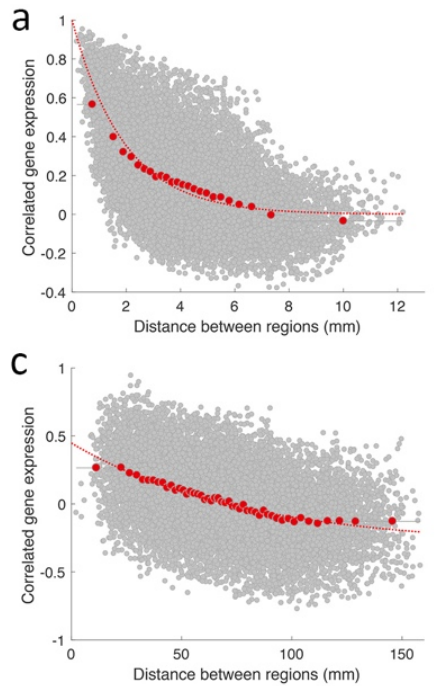

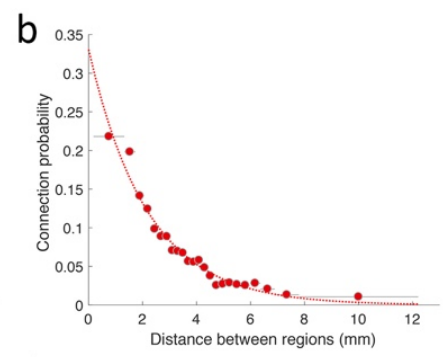

d

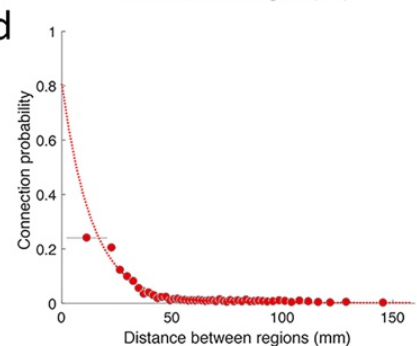

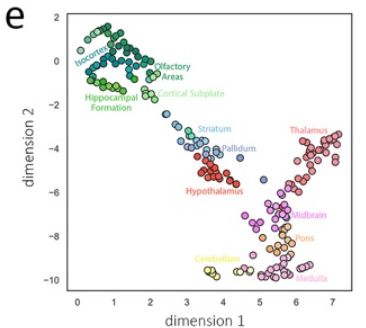

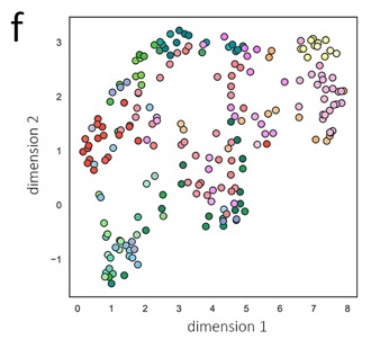

g

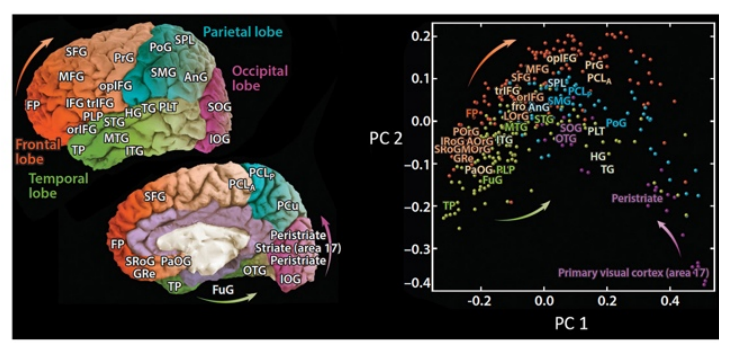

Figure 1. The brain transcriptome follows broad spatial trends that track brain network architecture. In the human cortex, both (a) CGE and (b) the probability of finding a connection between a pair of regions using diffusion MRI decline, as a function of their separation distance. Red line represents a fitted exponential trend. The mouse brain exhibits a similar distance-dependence of both inter-regional (c) CGE and (d) connection probability, as measured with anterograde tract-tracing. (e-f) Two-dimensional umap projections [128] of mouse brain regions based on their (e) gene transcription patterns (across 4211 genes from [18] passing our quality control criteria), and (f) axonal connectivity patterns (symmetrized version of connectome reported in [129] at $p<0.05$ significance threshold). Brain regions are coloured the same way in panels e and f. Regions in similar anatomical divisions cluster together in both representations, most prominently in the expression data. $(\mathrm{g})$ The first two principal components of expression measures in the AHBA segregate broad anatomical divisions in the human brain. Left shows the anatomical location of the colour scheme used in the right. Panels c-f adapted from data analysed in [31] and panel g reproduced from [45] based on data first presented in [19], with permission.

The distance-dependence of CGE and connection probability contributes to transcriptomic and connectomic differences both between and within large-scale anatomical divisions of the brain, and may underlie their functional and anatomical specialization [19,34-39] (Figure 1e-g). In humans, transcriptional differences between large-scale anatomical divisions (e.g., cortex vs cerebellum) are much greater than the differences within a division (e.g., between different cortical areas) $[19,40]$. The degree to which finer-grained subregions within an anatomical division possess distinctive transcriptional signatures is an open questionwhile there is some evidence for discrete transcriptional boundaries distinguishing functional subdivisions of hippocampus [41] and striatum [42], such boundaries have been difficult to identify in neocortex outside of 
primary sensorimotor areas $[19,43,44]$ (for a review, see [45]). A more consistent finding in neocortex is that expression patterns follow broad spatial gradients.

One prominent gradient of gene expression observed in the adult brain follows the rostrocaudal axis $[19,43]$, and is consistent with a similar transcriptional gradient observed prenatally $[39,46-48]$. This spatial pattern may be tied to regional variations in cellular architecture, intrinsic circuitry and extrinsic connectivity. Cortical areas show a prominent variation in neuron density and number along the rostrocaudal axis, such that both are higher in posterior regions $[49,50]$. This gradient is counteracted by a trend for increased neuronal size, arbor volume, and spine count in anterior areas, leading to more elaborate connectivity — both at the level of local microcircuitry and inter-regional connectivity-as one moves rostrally [32,49,51] (Figure 2a-b). The disparity in cortical microstructure between anterior and posterior areas is greater in species with larger brains, is thought to contribute to interspecies variation in brain size, and may explain the human advantage for abstract cognitive processes mediated by the frontal lobe [50-55]. These findings accord with twin modelling of human MRI data showing a strong rostrocaudal gradient of genetic influence on neocortical surface area expansion [56], and evidence that increasingly abstract cognition is supported by more anterior regions [57]. These rostrocaudal variations in cellular composition and connectivity are thought to arise from a corresponding and highly conserved spatial gradient in neurogenesis, which results in prolonged production, and ultimately density, of neurons in posterior regions; conversely, earlier termination of neurogenesis in anterior regions is thought to enable prolonged elaboration and consolidation of synaptic connectivity [49](reviewed in [51])(Figure 2a-b).
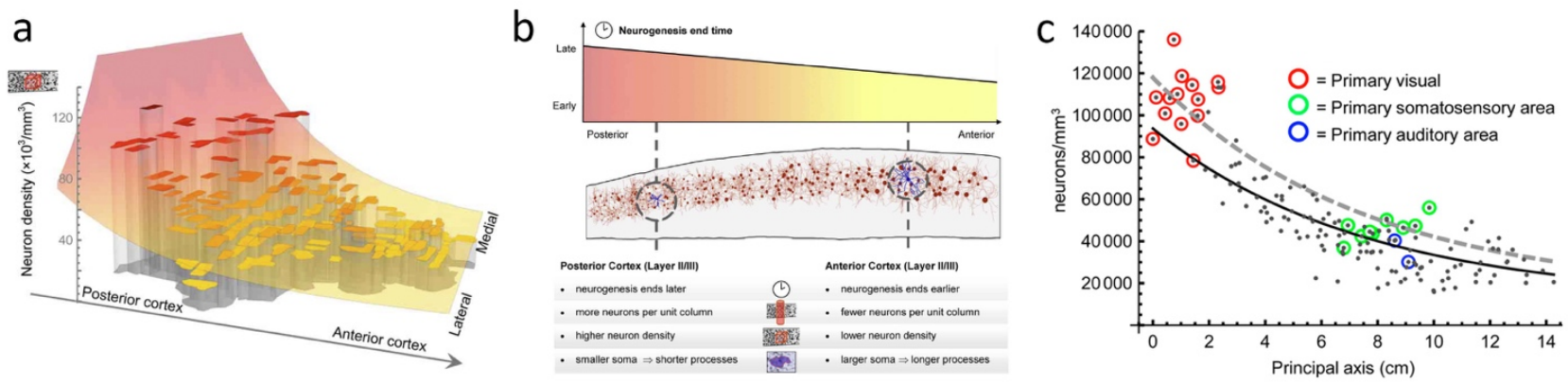

Figure 2. Neuronal density and synaptic connectivity vary along a rostrocaudal axis. (a) Empirical variations in regional neuron density across the baboon brain. Bars represent measures of neuron density taken from different tissue samples, situated along rostrocaudal and mediolateral axes. Bar heights correspond to the neuron density of each sample. The surface represents a mathematical function defining a global trend in neuron density that follows an axis extending from anterolateral to posteromedial cortex. Similar trends were observed in the macaque and galago. (b) Summary of the cellular and connectional properties that vary along the rostrocaudal gradient, as identified in mammalian brains. (c) Regional neuron density of the baboon cortex as a function of position along the rostro-caudal axis (lower numbers indicate more caudal locations). Solid line represents the fit of a single-factor mathematical model that characterizes variations only along this axis. The densities of primary sensory areas (coloured circles) are consistently higher than model predictions. A better fit is obtained when using a two-factor model that accounts for the rostrocaudal gradient and a distinction between primary and non-primary cortex (dotted line). Panels a and b reproduced from [49] and panel c from [54], with permission.

A simple mathematical model of rostrocaudal variations in neurogenesis can account for many aspects of cortical variations in neuron density across cortical areas and different species, but a two-factor model that includes a distinction between primary and non-primary cortex fits the data better [49,54](Figure 2c). This distinction between primary and other areas aligns with the well-known processing hierarchy of neocortical 
areas, which ranges from primary somatosensory to higher-order association areas, and is thought to reflect a progressive increase in the degree to which stimuli undergo integrated, multisensory processing [58-63] (Figure 3a-d). This hierarchical gradient is spatially correlated with the rostrocaudal axis (association areas are often found in frontal cortex), but its spatial patterning may diverge in species with expanded association cortex, such as humans, where the hierarchical gradient may assume a more prominent role in defining regional variations in cellular architecture and connectivity [64-66]. Consistent with this view, the principal gradient of regional variability in human functional connectivity closely tracks the functional hierarchy of cortical areas [58] (Figure 3d).

In macaques, hierarchical ordering of cortical areas has been quantitatively defined using laminar patterning of interareal projections, following observations that lower areas send mostly feedforward projections from supragranular layers to layer 4 neurons in higher areas, whereas higher-order areas send mostly feedback connections that originate in infragranular layers and terminate outside of layer 4 in lower regions [67-69] (Figure 3b). Accordingly, studies in the mouse, human, and non-human primate have identified substantial overlap between genes that show differential expression across cortical areas and those that exhibit layer-specific expression patterns $[43,70,71]$. Laminar patterning of gene expression is also closely tied to connectivity, with genes preferentially expressed in layers 3 to 5 being enriched in GO categories related to axons and neuron projections, a finding that may be driven by a higher density of long-range pyramidal projection neurons in these layers [43]. Moreover, interspecies differences in regional specialization and interregional connectivity may be driven by a small subset of genes with non-conserved laminar expression profiles $[43,45,71-73]$. Consistent with the last point, Krienen and Buckner [30] showed that CGE of 19 genes with enriched expression in layers 2-3 of human compared to mouse cortex distinguishes sensorimotor areas from limbic, paralimbic and association cortex. The average CGE of these genes is higher for regions possessing greater long-range compared to local functional connectivity, which is a signature of the integrative functions ascribed to association cortex. The authors thus propose that spatially patterned expression of HSE genes is a marker of the relative expansion of association cortex in the human brain $[64,74]$.

One limitation of defining cortical hierarchies based on laminar connectivity is that it requires invasive measurement. Recently, Burt and colleagues [66] showed in macaques that the contrast ratio of T1-to-T2 weighted MRI, which is sensitive to intracortical myelin content [75], correlates strongly with hierarchy inferred from laminar projection profiles (Figure 3c), suggesting that the ratio offers a non-invasive method for inferring a hierarchical ordering of cortical areas. In humans, the authors report a strong spatial correlation between the T1/T2 ratio and the first principal component brain-specific gene expression in the AHBA (Figure 3e). Genes known to be expressed in supra- and infra-granular layers showed increased expression along the hierarchy, whereas genes expressed in layer 4 showed decreased expression along the hierarchy. These findings parallel evidence that higher-order areas have less differentiated cortex and large soma, particularly in infragranular 
layers, which is thought to facilitate more efficient long-range connectivity [60] (Figure 3a). Burt and colleagues also found strong hierarchical gradients for transcriptional markers of distinct inhibitory cell types, NMDA receipt subunits and modulatory receptors. Consistent with these results in human cortex, the T1/T2 ratio of mouse cortex spatially correlates with regional variations in interneuron cell density, long-range axonal connectivity, and the expression of brain-specific genes [65]. Orthologous genes between mouse and human show a similar ordering in their strength of spatial correlation between transcription levels and $\mathrm{T} 1 / \mathrm{T} 2$, supporting a strong conservation of the hierarchical expression gradient.

a

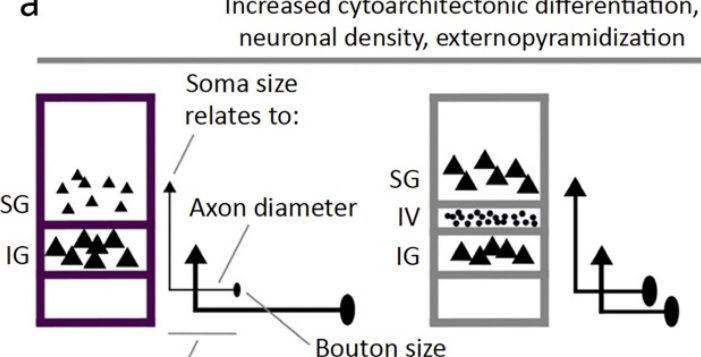

Axon length

Increased cytoarchitectonic differentiation, neuronal density, externopyramidization

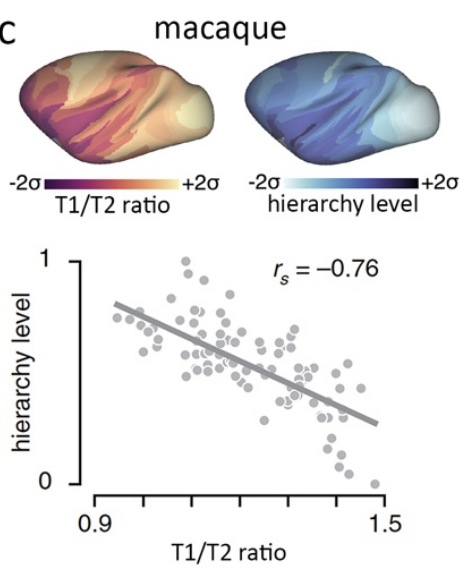

d
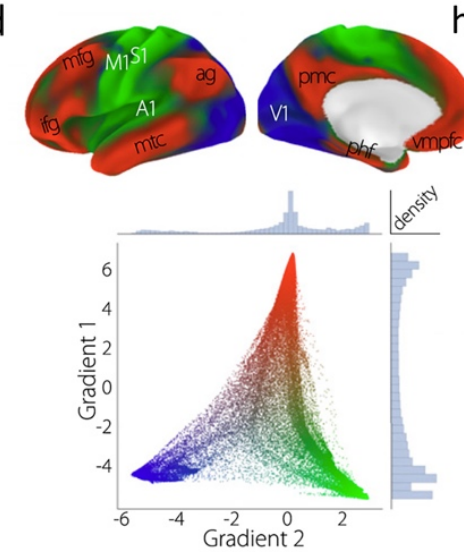

b

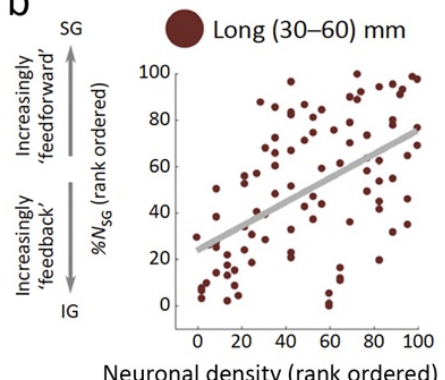

Neuronal density (rank ordered)
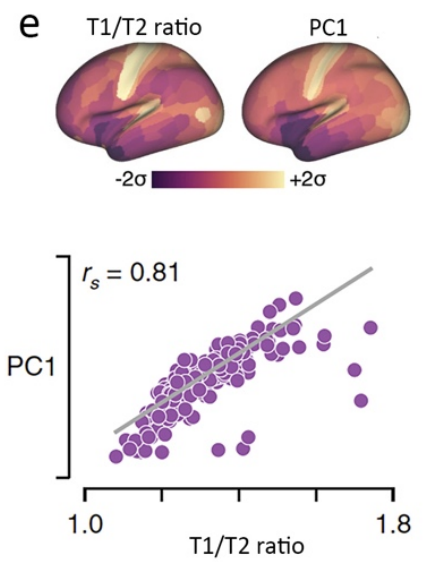

Figure 3. Hierarchical gradients in gene expression and connectional architecture. (a) The neocortical hierarchy is associated with numerous variations in cytoarchitecture. Moving down the hierarchy from association to primary cortex, there is a trend for greater cytoarchitectonic differentiation, higher neuronal density, and increased somal size of supragranular neurons compared to infragranular neurons. This trend in somal size variation, referred to as externopyramidization [60], is accompanied by corresponding shifts in average axon dimeter and synaptic bouton size. (b) Long-range (30-60 $\mathrm{mm}$ ) projections emanating from association areas, which have lower neuronal density, send proportionally more feedback projections, as quantified by the percentage of supragranular layers labelled by a retrograde tracer injected to that area (\% $N_{S G}$ ). (c) The ratio of T1-to-T2-weighted MRI signal tracks variations in myelin content (top left) and correlates strongly with hierarchy level, quantified using a measure derived from $\% N_{S G}$ (top right, bottom). (d) The two principal spatial gradients of human cortical functional connectivity, as identified via nonlinear dimensionality reduction. Bottom is a scatterplot situating each of thousands of cortical sites in the 2D space defined by the two gradients. The principal gradient, accounting for most of the variance (Gradient 1), separates primary sensorimotor areas (blue and green) from association, limbic and paralimbic cortices (red). Gradient 2 separates visual (blue) from somatomotor and auditory cortices (green). Top images colour anatomical locations according to the same gradients. (e) Spatial variations in the T1/T2 MRI ratio in humans are highly correlated with the first principal component of brainexpressed genes. Top shows the anatomical correspondence and bottom shows a scatterplot of the association across regions. Panels a-b reproduced from [60] and panels $c$ and e from [66], and d from [58] with permission.

Together, these brain-wide analyses linking connectome and transcriptome indicate that rostrocaudal and hierarchical spatial gradients of gene expression, functional specialization and inter-regional connectivity are closely related. In particular, genes showing hierarchical patterning tend to have layer-specific expression patterns, regulate axon formation and synaptic function, and relate to distinct cell and receptor sub-types, 
suggesting that they define a transcriptional signature of cortical microcircuit and macrocircuit specialization [64-66].

\section{Gene expression carries information about pairwise brain connectivity}

Do more specific relationships between gene expression and brain connectivity exist beyond the broad spatial gradients that dominate the brain's transcriptional landscape? Sperry's seminal experiments conducted over 70 years ago, which identified a remarkable specificity of axonal wiring over long anatomical distances, led him to propose that axons find their targets via the precise specification of molecular cues that link source and target neuron: the so-called chemoaffinity hypothesis [76]. This view suggests that pairs of connected neural elements may show a highly organized pattern of coupled gene expression. A large body of subsequent work has focused on identifying the specific molecules that guide axons to establish synaptic connections with appropriate partners, identifying important roles for molecules such as netrins, slits, ephrins, and semaphorins [77]. Some of these and other guidance molecules are indeed expressed along gradients, although theoretical considerations and experimental results suggest that these gradients are but one class of several distinct cues that are integrated by developing axons as they navigate to their targets [78].

Relationships between gene expression and connectivity that exist beyond broad spatial trends have been investigated either by statistically controlling or removing these trends from the transcriptional data $[31,32,79,80]$, or by constructing spatially constrained null models for appropriate statistical inference $[66,81,82]$. Using these approaches, it has been shown that average CGE across the genome is higher for connected compared to unconnected node pairs, defined at the level of mesoscopic brain regions in the mouse [31,32] (Figure 4a). Similar findings have been reported at the cellular level in C. elegans [33] (Figure 4b) and at the macroscopic level in human cortical morphometric covariance networks $[81,83]$. Genes contributing to increased CGE for connected regions in the mouse were enriched for $\mathrm{GO}$ categories related to axon development and maintenance, synapse formation, function and plasticity, and cellular metabolism. The genes most strongly implicated in elevated CGE for connected neurons in C. elegans included genes regulating gap junctions, cell adhesion, migration, and axon guidance. 

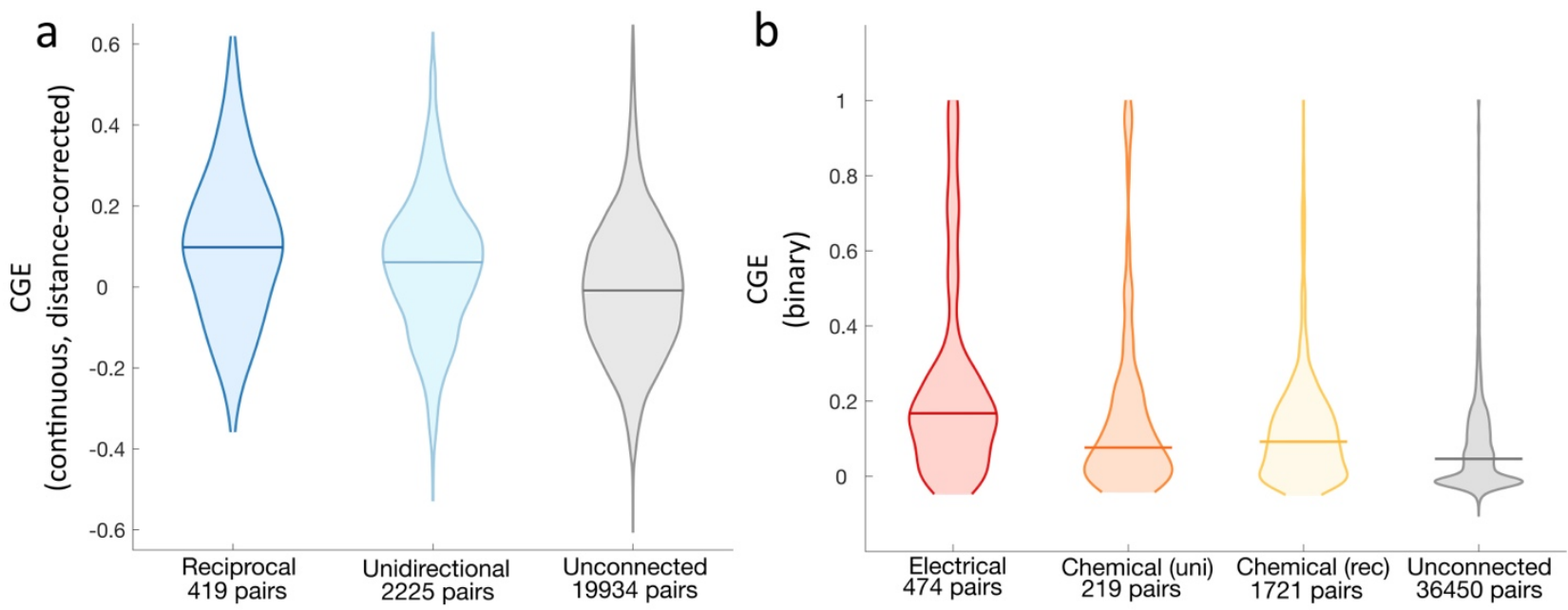

Figure 4. Transcriptional coupling is higher in anatomically connected compared to unconnected connectome elements. (a) Distance-corrected CGE in the mesoscale mouse connectome, as a function of connection type. CGE is higher for connected compared to unconnected regions, and is higher for reciprocally connected compared to unidirectionally connected pairs (all differences are statistically significant; Welch's t test, $p<10^{-6}$ ). (b) CGE for pairs of neurons in C. elegans linked by different types of connections; namely, electrical gap junctions, unidirectional (uni) chemical synapses, and reciprocal (rec) chemical synapses. As in the mouse (panel $a$ ), the CGE distribution is higher for connected compared to unconnected pairs of neurons ( $p=1.8 \times 10^{-78}$, Wilcoxon rank-sum test). CGE was quantified using the mean-square contingency coefficient due to the binary expression data available for $C$. elegans, and was not corrected for distance-dependence due to spatially complex relationships in the worm nervous system (see [33]). Panels a and b adapted from [31] and [33], respectively, with permission.

Several other studies have investigated whether gene expression patterns can predict the presence of a connection between pairs of neural elements [38,79,80,84-86]. In C. elegans, trained classification models are able to predict the existence of a synapse using expression patterns measured across $<300$ genes with an area under the curve (AUC) of 0.84 [85], and the incoming and outgoing connectivity partners of individual neurons with an AUC of 0.60 [86]. Gene expression profiles can also predict patterns of interregional axonal connectivity of the mesoscale mouse connectome with accuracies exceeding those of models based on interregional distance alone $[38,79,80]$ (see also [87]). Genes contributing strongly to these predictions are enriched in GO categories related to synapse and axon formation.

Together, these findings suggest that the brain transcriptome contains information about connectivity patterns that cannot be solely attributed to broad spatial trends. This conclusion aligns with a recent study reporting that CGE, in combination with spatial distance and structural network topology, best explained pairwise functional connectivity of the mouse brain, accounting for $62 \%$ of the variance [88]. A subset of 568 genes mainly annotated to GO categories related to potassium and cation channel activity made the strongest contributions to the effect of CGE. However, inter-regional distance was still the best individual predictor of functional connectivity, alone explaining up to $55 \%$ of the variance.

In humans, axonal connectivity is inferred indirectly through diffusion tractography, which has known spatial biases that affect the reconstruction of long tracts (e.g., [89]). Accordingly, results linking tractographic measures of connectivity to the AHBA have been less consistent than those in the worm and mouse. One study found a weak association between CGE and pairwise structural connectivity [36], while a later study reported a stronger association between seed region structural connectivity profiles and specific eigengenes of gene coexpression [35]. Studies of functional connectivity are less prone to the biases of diffusion tractography (but 
possess their own biases; e.g., [90]). One study found that coupled expression of genes with high differential stability is associated with stronger functional connectivity of cortical areas, and that genes with high differential stability are enriched for GO categories related to axon projections and guidance [40]. Wang et al. [91] found correlations between expression measures quantified using RNAseq across over 50 cortical samples and a regional measure of the amplitude of spontaneous low-frequency fMRI signal fluctuations, with a subset of 26 genes explaining $10 \%$ of signal amplitude variance in regions of the default mode network. Evidence in anesthetised mice suggests that spontaneous low-frequency fMRI signal fluctuations are strongly correlated with the total weight of axonal inputs to an area [92]. Thus, both these findings using human fMRI may be identifying transcriptomic correlates of the underlying structural connectivity between regions.

In summary, while broad spatial trends may explain most of the variance in structural connectivity and correlated transcriptional activity, the two quantities share specific information that cannot be explained solely by their spatial embedding. This additional information may be carried by a relatively small subset of genes involved in axon guidance and synapse formation, leading to the enrichment signatures observed in atlas-based analyses of the adult brain. These specific expression patterns likely complement broader spatial trends, such as those defining variations along rostrocaudal and hierarchical gradients, in shaping connectome structure and function.

\section{Transcriptomic signatures of connectome topology}

The striking conservation of topological properties observed across species and scales (Box 1) $[5,6]$ suggests that some of these properties are under genetic control, and should hence possess distinctive transcriptional signatures. These signatures have been studied most extensively in relation to topological substrates of functional segregation (modular architecture) and integration (network hubs and rich-club connectivity)(Box 1).

Richiardi et al. [93] first identified a link between gene expression and functional segregation, demonstrating that CGE was higher for regions within the same functional connectivity network of the human brain compared to regions in different networks. Genes driving this effect were enriched in GO categories related to ion channel function, and structural variants in these genes contributed to individual differences in functional connectivity. Elevated CGE for nodes within the same network has also been found for modules defined on human morphometric covariance networks [81] and on the neuronal connectome of C. elegans [33] (Figure 5a-b). While spatial biases may contribute to these findings, as nodes belonging to the same module tend to be anatomically colocalized $[7,8]$, they cannot explain these effects entirely $[94,95]$.

Several studies have examined transcriptional signatures of topological integration, focusing principally on the role of network hubs. Early work in the mouse and rat brains identified correlations between regional expression profiles and node degree and participation coefficient [32,96](see also [81]), but spatial autocorrelations in the 
expression data were not addressed. At the level of inter-regional transcriptional coupling, Fulcher and Fornito [31] fitted and removed the distance-dependence of CGE values (e.g., Figure 1a) and found that these distancecorrected CGE values were highest for connected pairs of network hubs (called rich links), intermediate for links between hubs and non-hubs (feeder links), and lowest for connected non-hubs (peripheral links) (Figure 5c-d). Hubs of the mouse connectome are separated by longer distances, on average, than other pairs of areas, so their elevated CGE contrasts with the general trend for CGE to decline with anatomical separation (e.g., Figure 1a). Genes driving elevated CGE for connected hubs are enriched in GO categories related to the oxidative synthesis and metabolism of adenosine triphosphate, whereas genes driving elevated CGE in connected compared to unconnected areas (see Figure 4a), regardless of hub status, were additionally enriched in categories related to axonal and synaptic structure and function. The strong involvement of metabolic genes in this putative transcriptional signature of hub connectivity aligns with evidence that brain network hubs mediate the lion's share of signal traffic in the brain and are among the most metabolically active elements of the connectome [7,97-100]. The findings also suggest that while the expression of genes regulating axonal and synaptic structure and function may distinguish connected from unconnected regions, the primary transcriptional distinction between different topological classes of connections in the mature brain may be related to the metabolic requirements of the regions that they connect.

More recently, elevated CGE between connected network hubs has also been found in the microscale connectome of $C$. elegans [33] (Figure 5e). This effect could not be attributed to similarities between hub neurons in terms of birth time, lineage distance, module affiliation, anatomical location, neuronal subtype or secreted neurotransmitter. Instead, the effect was attributable to the fact that all hub neurons in the worm correspond to a specific subclass of command interneurons that play a critical role in regulating locomotion, one of the most complex behaviours available to the animal. These findings thus parallel evidence that network hubs in mammals are predominantly located in association cortex, which mediates complex, integrative processing $[64,101]$.

The consistency of the CGE elevations for connected hubs across worm and mouse is notable given the different species (invertebrate vs mammal), orders of magnitude difference in analysis resolution (single cell in worm vs mesoscale regions in mouse), and major differences in expression data (binary expression data for 948 genes curated from published literature in the worm vs quantitative ISH measures for $>17,000$ genes in the mouse) $[31,33]$. Evidence from fMRI suggests that a comparable signature may also be present in humans. Using partial least squares, Vértes et al. [82] identified a latent component describing transcriptional variance in genes regulating oxidative metabolism that correlated with nodal participation coefficient, inter-module degree, and long-range connectivity, high levels of which are characteristic of network hubs [101]. 

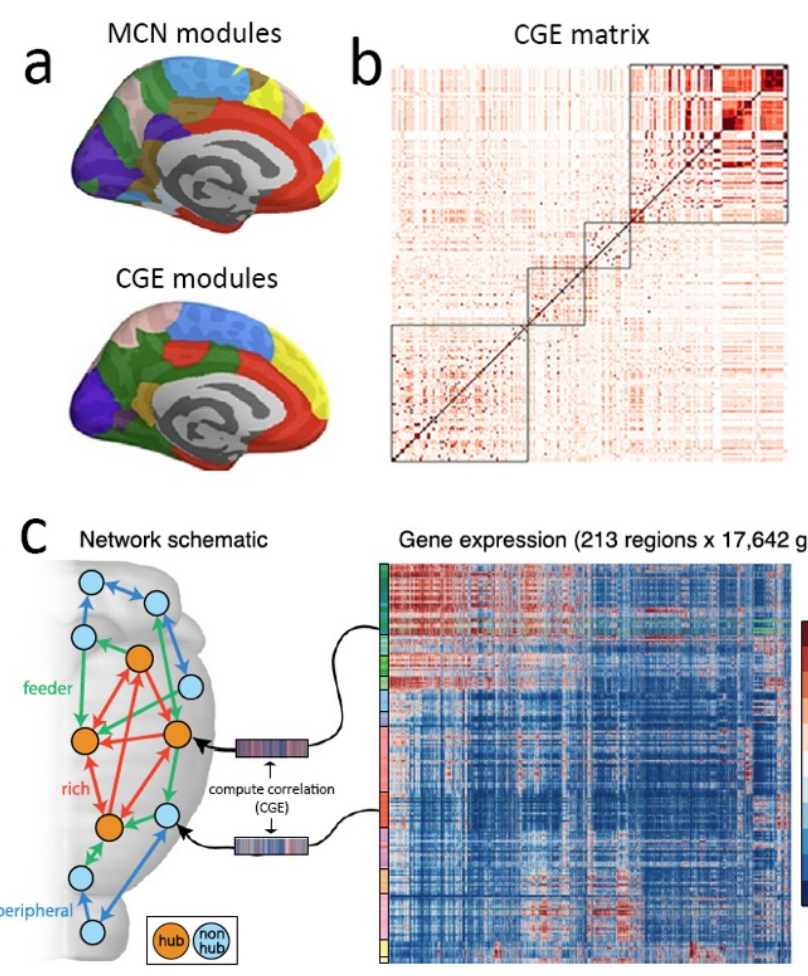

Gene expression (213 regions x 17,642 genes)

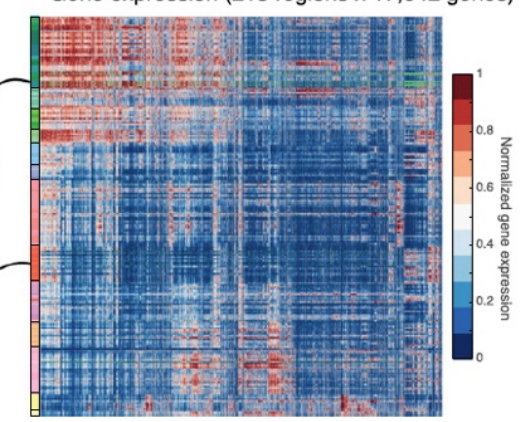

$\mathrm{e}$

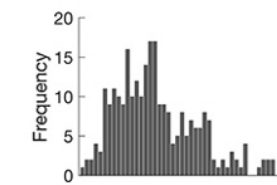

worm

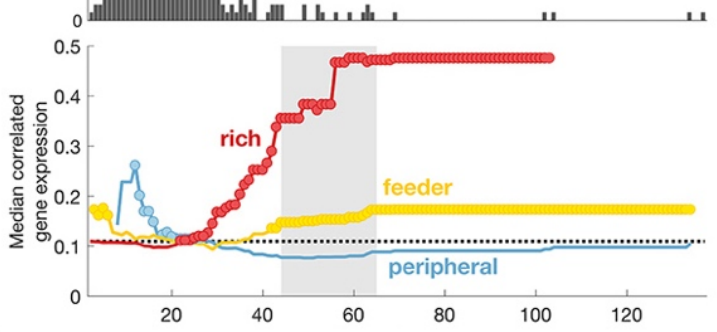

d

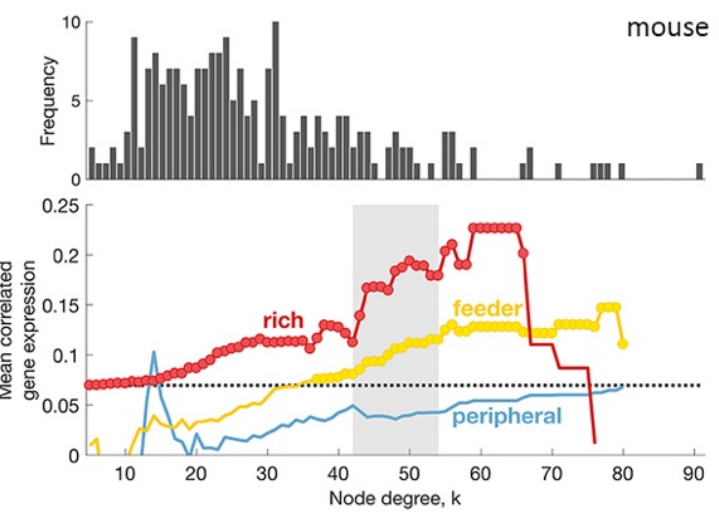

Figure 5. Transcriptional coupling is elevated within network modules and between network hubs. (a) The modules of a human region $\times$ region morphometric covariance network $(\mathrm{MCN})$ and region $\times$ region CGE network have a similar spatial topography. Different colours indicate module affiliations. (b) Matrix of CGE values for all pairs of neurons in Celegans, as quantified using the mean-square contingency coefficient. The matrix has been ordered by neuronal module affiliation, with module boundaries delineated by black squares. Average CGE is higher (dark red) is higher for connected pairs of neurons within the same topological module (neurons in the same box) (c) Schematic showing how hub connectivity can be related to CGE in the mouse brain. Nodes are classified as hubs or non-hubs using a specific degree threshold, allowing a topological distinction between three types of connections: rich (links between two hubs), feeder (links between hubs and non-hubs) and peripheral (links between two non-hubs) [109]. The corresponding expression vectors for any pair of regions can then be extracted from the gene expression matrix (right) to estimate their CGE. (d) Top panel shows the degree distribution for inter-regional connectivity in the mouse. The distribution has an extended tail, consistent with the existence of highly connected hubs. Bottom panel shows CGE for rich, feeder and peripheral links as a function of degree threshold for defining hubs. Higher values on the $x$-axis represent more stringent definitions of hubs. Grey area indicates a threshold regime showing topological evidence for rich-club organization (i.e., higher inter-connectivity between hubs than expected by chance). A sharp rise in CGE for rich links coincides with the onset of this topological rich-club regime. Dotted horizontal line shows mean CGE across all links. (e) Same data as presented in panel $d$ for the $C$ elegans connectome. As in the mouse, CGE is highest for rich links and increases sharply in the topological rich-club regime. Panel a reproduced from [81], panels c-d from [31], and panel e from [33] with permission.

Together, these findings point to transcriptional signatures of both modular organization and hub connectivity that are conserved across the worm, mouse and human. There appears to be some specificity in the genes driving these signatures, such that genes regulating ion channels may drive elevated CGE within functional connectivity networks [93], whereas genes regulating oxidative metabolism dominate the transcriptional signature of hub connectivity [31,82]. However, it is as yet unclear whether these apparent signatures of large-scale network topology are truly distinct. Given that hubs are strongly inter-connected, and may be viewed as comprising a distributed module themselves [102,103], elevated CGE between hubs may simply represent a specific example of a more general tendency for increased CGE within functionally related and interconnected neural systems. This conclusion is consistent with reports that HSE genes are implicated in the transcriptional signatures of both network modules and hubs $[81,82]$. Another open question concerns the overlap between these transcriptional signatures of network topology and hierarchical gradients, given that cortical hubs are predominantly found in association cortex [64,101]. 


\section{Concluding remarks}

The evidence reviewed here indicates that anatomical variations in gene expression closely track variations in connectional architecture. These variations are dominated by large-scale spatial gradients, upon which are superimposed more specific transcriptional signatures of network connectivity and topology.

The research considered here, comprising the first wave of studies linking brain-wide expression measures to connectome-wide phenotypes, has been encouraging insofar as the transcriptional correlates of the neural phenotypes examined are generally sensible and implicate genes that plausibly relate to neuronal connectivity. These findings support the utility of atlas-based approaches as a method for gaining insight into the transcriptional correlates of spatially varying neural phenotypes, both in health and disease (Box 4). Indeed, a strength of these approaches is that they can be easily applied to human imaging data (although see [23]) and related to atlases constructed in other species. However, an understanding of the specific molecular mechanisms underlying network organization requires investigations that extend beyond the correlational approaches deployed thus far (Box 3). Technical developments that improve the precision of both transcriptional and connectomic measures (Boxes 2,3) will undoubtedly enhance our capacity to unravel the molecular basis of large-scale brain network organization. 


\section{Glossary}

Candidate variant. A specific DNA variant chosen for further analysis (e.g., to test for an association with phenotypic variability). Effective connectivity. The causal (directed) influence that one neuronal system exerts over another.

Eigengene. The first principal component of expression variance for a subset of genes, where the subset is typically defined as a module of a gene coexpression network.

Expression quantitative trait loci. Genetic loci that impact the expression levels of a gene.

Default mode network. A network of brain regions including posterior cingulate, medial prefrontal and lateral parietal cortices.

Differential stability. The degree to which a gene exhibits a consistent regional expression profile across the six donor brains comprising the Allen Human Brain Atlas.

Functional connectivity. A statistical dependence between neurophysiological signals acquired from distinct neural elements.

Gene ontology. A hierarchical organization of terms (most commonly biological functions, but also molecular functions and cellular components), to which genes are constantly being annotated based on the latest scientific evidence.

Genome-wide association. Analysis approach that tests for associations between phenotypic variability and markers of allelic variation distributed throughout the genome.

Hierarchical modularity. A network module is a subset of densely interconnected nodes. Hierarchical modularity occurs when modules contain smaller submodules over several resolution scales.

Lineage distance. Number of divisions separating two cells from a common progenitor.

Morphometric covariance. Inter-regional statistical dependence in some measure of brain morphopmetry, such as regional volume, cortical thickness or microstructure.

Neotenous. A pattern of gene expression that persists through development into adulthood.

Network economy. The management of network resources (connectivity, activity, etc) to support robust, adaptive and costefficient performance.

Node degree. The number of connections attached to a network node.

Partial least squares. A multivariate method that explains a set of observed variables as a linear combination of a set of predictor variables.

Participation coefficient. The distribution of a node's connections across different topological modules of a network. Nodes with high participation have an equal distribution of edges across modules.

Rich club. A set of highly connected network nodes that are more strongly interconnected with each other than expected by chance.

Small-world. A network topology characterized by more clustered connectivity and comparable average shortest path length (the average minimum number of edges between nodes) relative to a random graph.

Topology. The topology of a network is the specific arrangement of pairwise connections between nodes, and is invariant to the physical embedding of the system.

Transcriptome. The complete set of RNA molecules in a cell(s). Some transcriptional assays may only sample a subset of these molecules.

Wiring costs. The metabolic cost of neuronal connectivity. Often approximated by the volume or distance of anatomical connections. 


\section{Box 1-Representing the brain as a complex network}

Any network, including the brain, can be represented as a graph of nodes connected by edges. In connectomics, the nodes can represent neurons, neuronal populations or macroscopic brain regions, and the edges represent some index of structural connectivity, functional connectivity, or effective connectivity (Figure la). The full set of connections in the network can be summarized in the form of a connectivity matrix, which is formally equivalent to a graph [4,5] (Figure Ib). By distilling a connectome to its essential elements--nodes and edges--we can conceptualize the brain as one example of a much broader class of complex systems [104]. The mathematical foundations of network science, which are grounded in graph theory and statistical physics, offer a rich repertoire of measures for characterizing diverse aspects of brain network topology and dynamics [4,5]. The application of these tools to connectomic data, acquired across different species and resolution scales, have identified a remarkable conservation of topological properties [6]. These properties include economical wiring, such that the total wiring volume of the network is near minimal relative to the topological complexity of the network [7,105]; hierarchical modularity and small-world organization [106,107]; and the presence of highly connected network hubs, which are also densely connected with each other, forming a rich club [108] (Figure Ic). Rich-club connections between hubs typically extend over long anatomical distances, connect disparate functional systems, and are positioned to mediate a high volume of network traffic, indicating that these costly and topologically central links play a vital role in integrated brain function [31,97,109,110]. Modular organization on the other hand, is thought to support specialization and segregation of function [111](Figure Ic).

Brain networks are intrinsically directed, weighted, and have heterogeneous nodes (e.g., brain regions vary in microstructural properties like cellular composition and cytoarchitecture) and edges (e.g., connections can be excitatory or inhibitory). Brain networks also evolve through time. Most analyses to date have relied on the simplest network representation: a static, undirected binary graph [5,112]. Improved measurement techniques that enhance our capacity to resolve directionality, connection weights, and heterogeneity in brain networks are yielding new insights into connectome topology [113-115], and will be important for providing a level of specificity that matches developments in transcriptional assays (Box 2).

\section{a}
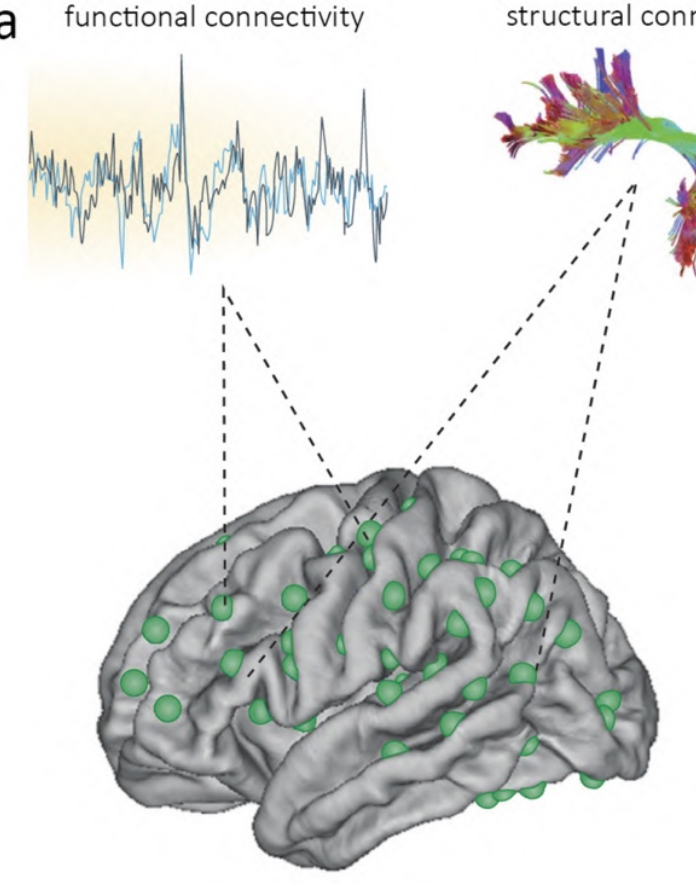

structural connectivity

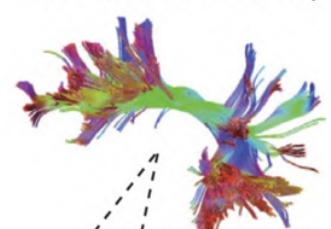

b

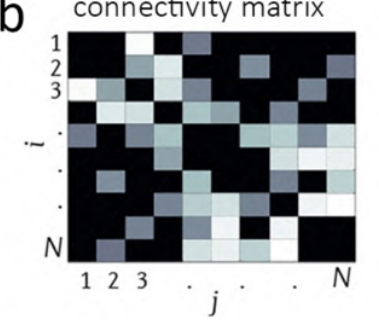

brain graph

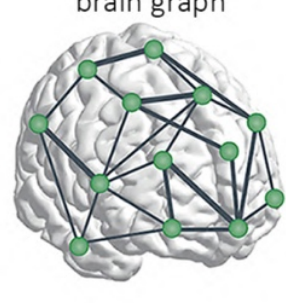

C network topology

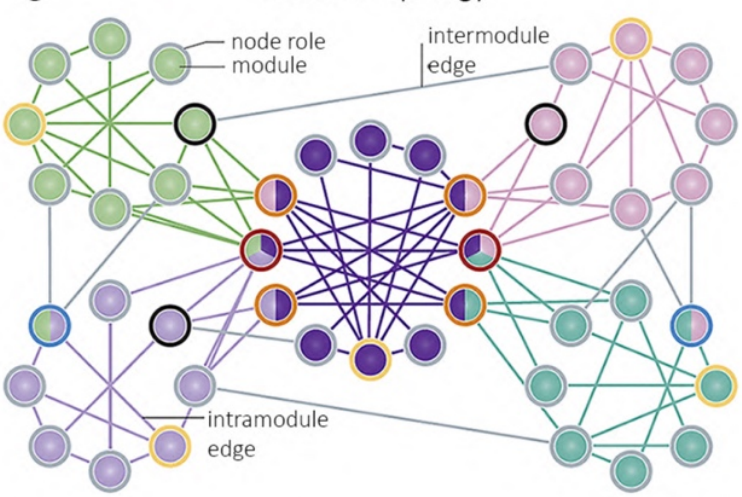

Figure I. Major steps in connectome construction and analysis. (a) The brain is parcellated into distinct regions, which act as network nodes (green). Functional or structural connectivity is then measured for each node pair (effective connectivity not shown). (b) Pairwise connectivity in the network can be succinctly represented as an $N \times N$ matrix, where $N$ is the number of nodes and each $[i, j]$ element encodes the connectivity between node pairs (left). Equivalently, the network can be represented in graph form (right). (c) The graph model can be used to characterize distinct aspects of network topology. In this hypothetical projection, nodes are clustered together based on similarity in connectivity, highlighting some key topological properties relevant to the brain. For instance, nodes cluster into modules (different colours), and some nodes participate in multiple modules (multicoloured nodes). Hubs can either be provincial, and highly connected within their own module (orange border) to support functional specialization, or act as connector hubs that link different modules (red border) to support functional integration. Hubs are strongly interconnected, forming a rich club. The rich club may also be viewed as a module (purple) that overlaps with other, more specialized modules. The rich club supports integration between topologically segregated modules. Figure adapted from [103] with permission. 


\section{Box 2 - Constructing and analyzing gene expression atlases}

Gene expression can be quantified in several ways $[16,18,19]$. The two techniques yielding the most anatomically comprehensive brain atlases thus far are in situ hybridization (ISH) and microarray. ISH was used to construct the AMBA [18,24], and allows subcellular localization of a specific segment of nucleic acid within an entire histologic section. Microarray was used to construct the AHBA [19] and simultaneously quantifies the expression levels of thousands of genes through hybridization of Cy3-labeled RNA in a tissue sample to a probe on a microarray chip that maps to a unique DNA sequence.

One limitation of microarray is that it is applied to bulk tissue samples that comprise an admixture of different cell types (for other limitations, see [116]). Thus, the expression signature of a given sample may be driven by its cellular composition. Single-cell RNAseq has emerged as a promising approach for cell-specific expression profiling [117]. Efforts are underway to improve the scalability of these and related techniques [16], but current single-cell transcriptomic atlases lack the anatomical coverage of the AMBA and AHBA (for a review of available atlases, see [20]).

The measures comprising the AMBA, AHBA and other atlases are subjected to rigorous quality control procedures, but further processing of the data is often required prior to analysis. Choices at each processing stage can impact the final results and should be made carefully (for further details, see [23]). The transcriptomic measures are then typically analyzed at the level of (1) regional gene expression; (2) correlated gene expression (CGE) between neural elements; (3) or gene coexpression networks (Figure II). Analyses of regional expression aim to identify associations between regional variations in gene expression and some regional property, such as node degree [32] (Figure IId). Analyses of CGE examine how transcriptional coupling between regions relates to pairwise attributes of network organization, such as whether two regions are connected or not, or the type of connection they share [31-33,79,80,84-87] (Figure Ile). Analyses of gene coexpression networks consider correlations in the regional expression profile of pairs of genes (e.g., $[35,118]$ ) (Figure IIc). Thus, while CGE focuses on region-to-region coupling across genes, gene coexpression analyses focus on gene-to-gene coupling across regions (Figure IIc,e). Note that this terminology is not used consistently, and some studies refer to CGE as coexpression. We use this specific nomenclature here to minimize confusion.

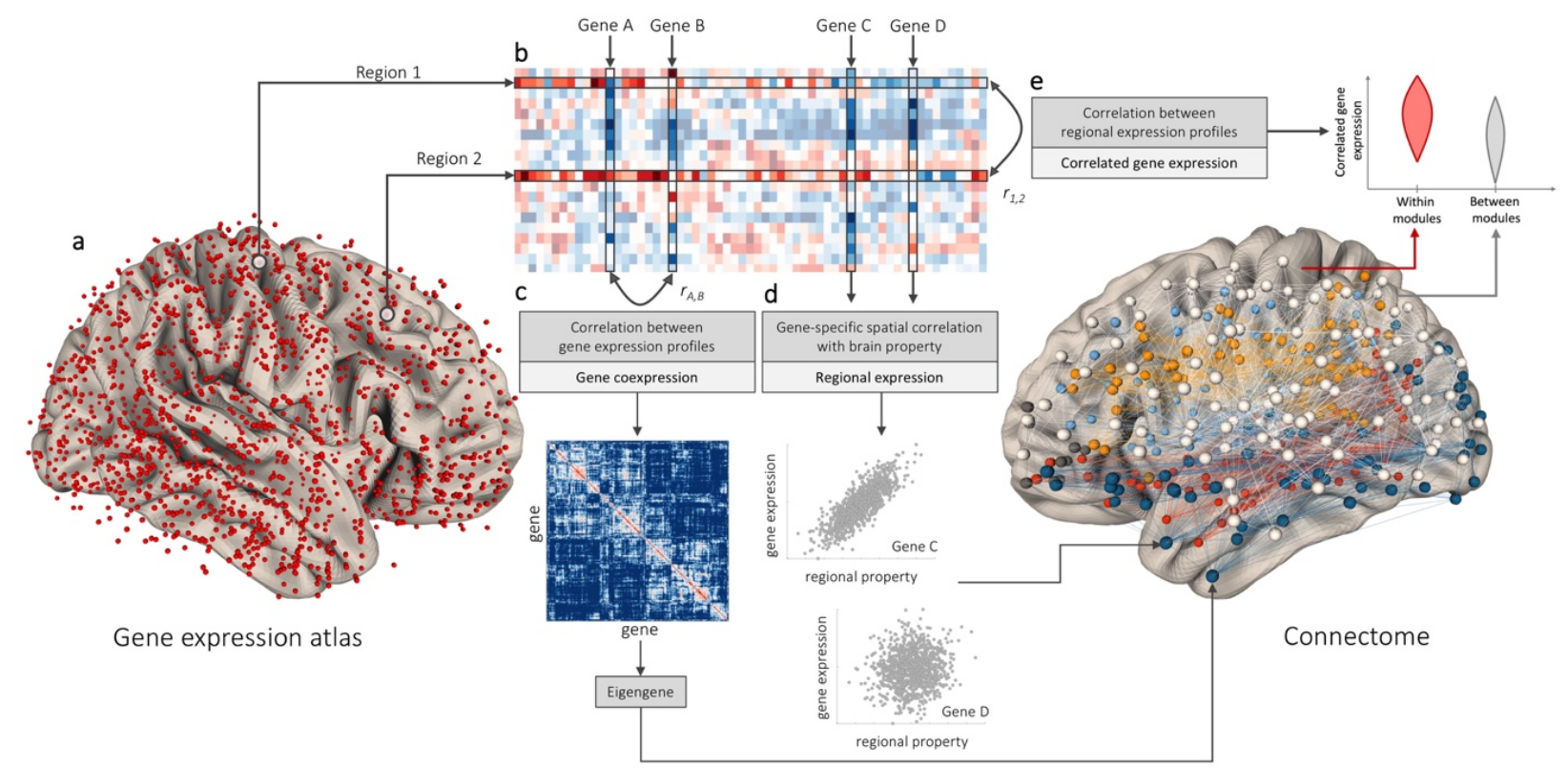

Figure II. Schematic of basic workflows for relating spatial variations in gene expression to brain network properties. (a) Brain surfaces show the locations of different cortical tissue samples taken from the six donor brains comprising the AHBA (left) and an example connectome constructed using diffusion MRI data (right), where colours indicate node membership to different topological modules. (b) Each sample in the expression atlas is characterised by a vector of expression values across genes. These region-specific vectors can be combined into a region $\times$ gene expression matrix. (c) Gene coexpression is the correlation between the regional expression profiles of pairs of genes (i.e., columns of the expression matrix). It can be estimated for each pair of genes to obtain a gene coexpression matrix. Summary measures of expression patterns in this matrix, such as eigengenes, can then be mapped back onto the brain and related to regional properties of network organization. (d) Analysis of regional expression patterns involves extracting each gene's spatial profile separately and correlating it with regional variations in some brain property (e.g., volume, activation, node degree). In this hypothetical example, Gene C shows a spatial correlation with the brain property whereas Gene D does not. (e) In a CGE analysis, vectors of gene expression for each region (rows of the expression matrix) are correlated with each other. CGE values can then be related to pairwise measures of brain structure or function. In this hypothetical example, the distribution of CGE values for connections within modules is compared to CGE values for connections between modules. 


\section{Box 3 - Limitations and future prospects of brain-wide transcriptomics}

Most studies linking brain-wide gene expression and imaging data rely on correlational methods (Box 2). This correlational approach cannot, in isolation, reveal the molecular mechanisms of a given neural phenotype, but it can prioritize genes for further study. Prioritization is important given our (presently) limited understanding of how genes contribute to large-scale connectome properties. In model species, the priority list can then be interrogated with more invasive methods (e.g., [17]). In humans, allelic variability in the priority genes can be related to expression quantitative trait loci and phenotypic variability across individuals, thereby identifying specific DNA variants that impact gene transcription and phenotype expression $[93,119]$. An advantage of prioritizing genes through atlas-based approaches is the smaller multiple comparison burden when compared to a naïve genome-wide search for allelic associations ( 20,000 genes vs $\sim 1$ million variants). Atlas-based analyses can also clarify the phenotypic effects of putative disease risk genes (Box 4.)

One caveat to atlas-based analyses is that the most comprehensive atlases currently available assay expression in the adult brain. Some genes expression patterns are neotenous [120], but the vast majority (i.e., >80\%) show differential expression across development [121]. The extension of developmentally-resolved transcriptome atlases [121,122], and their linkage to imaging data acquired across the lifespan (e.g., [74,123]), will shed light on how transcriptional dynamics relate to brain network maturation and ageing.

A second caveat is that most current atlases do not delineate cell-specific expression patterns. Single-cell transcriptomics is revealing a tremendous diversity of genomic signatures related to cellular morphology, physiology and connectivity (e.g., [124]). This diversity is lost when expression levels are aggregated across cell types within a bulk tissue sample. Improvements in the scalability of single-cell profiling will yield a new generation of atlases with unprecedented precision [16].

A third caveat is the reliance on enrichment analysis in most atlas-based studies. Enrichment analysis is a useful strategy for gaining preliminary insight into the transcriptional correlates of a given connectome property while mitigating the multiple comparison burden, but many GO categories comprise scores of genes so more focused follow-up investigations are required to identify which specific genes are most relevant to the phenotype. Finally, connectome properties are often quantified in a different set of individuals to those used to construct the expression atlas. Transcriptional variance is much greater across brain regions than across individuals [19], but our understanding of how spatial patterns of gene expression vary across individuals is limited. 


\section{Box 4-Clinical applications of brain-wide gene expression atlases}

Brain imaging is widely used to map the changes in neural structure and function that associate with a particular clinical disorder, but the molecular mechanisms causing these macroscopically identified changes are often unclear. Brain-wide gene expression atlases are increasingly being used to gain insight into such mechanisms (for caveats, see Box 3).

The most general approach involves testing for correlations between the spatial topography of group differences in some imaging phenotype and the spatial expression pattern of each gene. For example, partial least squares has been used to identify components of genetic variance with expression patterns that correlate with the topography of cortical thickness changes in autism spectrum disorder [81] (Figure IIla-c) and structural connectivity reductions in Huntington disease [125]. In both cases, many of the genes linked to the imaging phenotypes were found to have altered expression in patient brains examined post-mortem. This correspondence suggests that spatial correlations between transcriptional patterns in the AHBA and case-control differences in imaging measures can be used to identify pathologically relevant genes.

A more focused approach involves using an expression atlas to elucidate the phenotypic effects of disease risk genes. In schizophrenia, the spatial pattern of regional reductions in structural connectivity strength correlates strongly with the spatial pattern of the average expression of disease risk genes identified through genome-wide association [126] (Figure IIId-e). No comparable spatial correlation was observed between gene expression and cortical thickness changes, suggesting that the effects of these genes on illness liability are mediated through inter-regional connectivity rather than regional morphometry. A similar approach in Parkinson disease found that the spatial expression pattern of the tau gene MAPT correlated with the topography of connectivity reductions in patients [127]. No such association was observed for the SNCA gene encoding alpha-synuclein, indicating that macroscale network changes in the disorder may be mediated primarily through tau pathology.

In a third approach, genes with expression patterns tied to normative imaging phenotypes are tested for overlap with known risk genes for a given disorder (e.g., [31,32,87,91]). For instance, Whitaker et al. [123] identified a component of genetic variance correlated with developmental changes in cortical thickness and myelination during healthy adolescence. Genes loading on this component were enriched in GO categories related to synaptic transmission, and glutamatergic and ion channel signalling, and were over-represented in risk genes for schizophrenia. These findings thus establish a link between genetic mechanisms of schizophrenia risk and normal brain maturation.
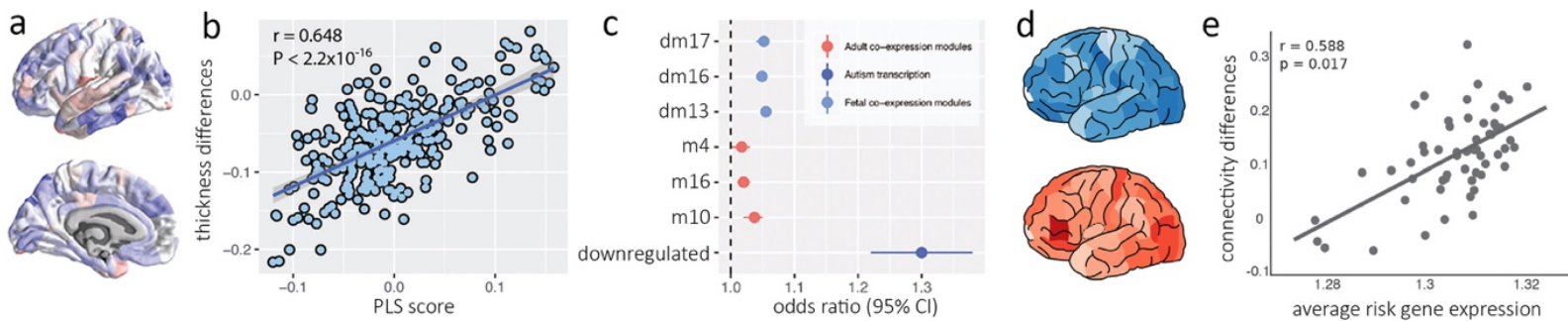

Figure III. Linking gene expression to brain changes in clinical disorders. (a) Spatial topography of cortical thickness differences between children with autism and healthy controls (blue thicker in controls; red thicker in autism). (b) Scatterplot of the spatial correlation between autism-control thickness differences and regional loadings on a partial least square (PLS) component of transcriptomic variance showing enrichment for the GO category "synaptic transmission". (c) Odds ratios for an enrichment analysis of PLS scores in relation to different modules of gene coexpression networks that are downregulated in patients' brains (see [81] for further details). The results depicted in (d) and (e) were replicated in three samples. We show here data from the sample showing the strongest association between PLS scores and thickness differences (weakest observed was $r=$ $0.32, p=4.15 \times 10^{-9}$ ). (d) Spatial topography of the average expression of 48 risk genes for schizophrenia identified through genome-wide association (top; darker blue is higher expression) and topography of patient reductions in regional structural connectivity (bottom; darker red is greater reduction in patients). (e) Scatterplot of the spatial correlation between regional variations in average risk gene expression and group differences in structural connectivity for schizophrenia. Panels a-c reproduced from [81] and panels d-e from [126] with permission. 


\section{Acknowledgements}

AF was supported by The Charles and Sylvia Viertel Foundation, Australian Research Council (FT130100589) and National Health and Medical Research Council (NHMRC; IDs: 1146292, 1104580, and 1066779). BDF was supported by an NHMRC Fellowship (1089718). 


\section{References}

1 Sporns, O. et al. (2005) The human connectome: A structural description of the human brain. PLoS Comp Biol 1, e42

White, J.G. et al. (1986) The structure of the nervous system of the nematode Caenorhabditis elegans. Philos Trans R Soc Lond B Biol Sci 314, 1-340 Hagmann, P. et al. (2008) Mapping the structural core of human cerebral cortex. PLoS Biology 6, e159 Bullmore, E. and Sporns, O. (2009) Complex brain networks: graph theoretical analysis of structural and functional systems. Nat Rev Neurosci 10, 186-198 Fornito, A. et al. (2016) Fundamentals of Brain Network Analysis, Academic Press, Inc. van den Heuvel, M.P. et al. (2016) Comparative Connectomics. Trends Cogn Sci DOI: 10.1016/j.tics.2016.03.001

Bullmore, E. and Sporns, O. (2012) The economy of brain network organization. Nat Rev Neurosci 13, 336-349

Henderson, J.A. and Robinson, P.A. (2011) Geometric effects on complex network structure in the cortex. Phys Rev Lett 107, 018102

Ercsey-Ravasz, M. et al. (2013) A Predictive Network Model of Cerebral Cortical Connectivity Based on a Distance Rule. Neuron 80, 184-197 Betzel, R.F. et al. (2016) Generative models of the human connectome. Neurolmage 124, 1054-1064 Gămănuţ, R. et al. (2018) The Mouse Cortical Connectome, Characterized by an Ultra-Dense Cortical Graph, Maintains Specificity by Distinct Connectivity Profiles. Neuron 97, 698-715.e10 Fornito, A. et al. (2011) Genetic Influences on Cost-Efficient Organization of Human Cortical Functional Networks. J Neurosci 31, 3261-3270 van den Heuvel, M.P. et al. (2013) Genetic control of functional brain network efficiency in children. Eur Neuropsychopharm 23, 19-23 Glahn, D.C. et al. (2010) Genetic control over the resting brain. Proc Natl Acad Sci USA 107, 1223-1228 Smit, D.J. et al. (2008) Heritability of "small-world" networks in the brain: a graph theoretical analysis of resting-state EEG functional connectivity. Hum Brain Mapp 29, 1368-1378

Lein, E. et al. (2017) The promise of spatial transcriptomics for neuroscience in the era of molecular cell typing. Science 358, 64-69 Lein, E.S. et al. (2006) Genome-wide atlas of gene expression in the adult mouse brain. Nature 445, 168176

Hawrylycz, M.J. et al. (2012) An anatomically comprehensive atlas of the adult human brain transcriptome. Nature 489, 391-399

Keil, J.M. et al. (2018) Brain transcriptome databases: a user's guide. I Neurosci DOI: 10.1523/JNEUROSCI.1930-17.2018

Liu, Y. et al. (2016) On the Dependency of Cellular Protein Levels on mRNA Abundance. Cell 165, 535550

Maier, T. et al. (2009) Correlation of mRNA and protein in complex biological samples. FEBS Letters 583, 3966-3973

Arnatkeviciute, A. et al. (2018) A practical guide to linking brain-wide gene expression and neuroimaging data. bioRxiv DOI: 10.1101/380089

\section{2}

$\mathrm{Ng}$, L. et al. (2009) An anatomic gene expression atlas of the adult mouse brain. Nat Neurosci 12, 356-

25 Ashburner, M. et al. (2000) Gene ontology: tool for the unification of biology. The Gene Ontology Consortium. Nat Genet 25, 25-29

26 Ogata, H. et al. (1999) KEGG: Kyoto Encyclopedia of Genes and Genomes. Nucleic Acids Res. 27, 29-34

27 Rhee, S.Y. et al. (2008) Use and misuse of the gene ontology annotations. Nature Reviews Genetics 9, 509-515

28 Tomczak, A. et al. (2018) Interpretation of biological experiments changes with evolution of the Gene Ontology and its annotations. Sci Rep 8, 5115 
Uygun, S. et al. (2016) Utility and Limitations of Using Gene Expression Data to Identify Functional Associations. PLoS Comp Biol 12, e1005244

30 Krienen, F.M. et al. (2016) Transcriptional profiles of supragranular-enriched genes associate with corticocortical network architecture in the human brain. 113, E469-E478

31 Fulcher, B.D. and Fornito, A. (2016) A transcriptional signature of hub connectivity in the mouse connectome. Proc Natl Acad Sci USA 113, 1435-1440

French, L. and Pavlidis, P. (2011) Relationships between Gene Expression and Brain Wiring in the Adult Rodent Brain. PLoS Comp Biol 7, e1001049-12

Arnatkeviciute, A. et al. (2018) Hub connectivity, neuronal diversity, and gene expression in the Caenorhabditis elegans connectome. PLoS Comp Biol 14, e1005989-32

Bohland, J.W. et al. (2010) Clustering of spatial gene expression patterns in the mouse brain and comparison with classical neuroanatomy. Methods 50, 105-112

Forest, M. et al. (2017) Gene networks show associations with seed region connectivity. Hum Brain Mapp 38, 3126-3140

Goel, P. et al. (2014) Spatial patterns of genome-wide expression profiles reflect anatomic and fiber connectivity architecture of healthy human brain. Hum Brain Mapp 35, 4204-4218

Song, S. and Abbott, L.F. (2001) Cortical development and remapping through spike timing-dependent plasticity. Neuron 32, 339-350

Fakhry, A. et al. (2017) Global analysis of gene expression and projection target correlations in the mouse brain. Brain Informatics DOI: 10.1007/s40708-015-0014-2

Ko, Y. et al. (2013) Cell type-specific genes show striking and distinct patterns of spatial expression in the mouse brain. Proc Natl Acad Sci USA 110, 3095-3100

40 Hawrylycz, M. et al. (2015) Canonical genetic signatures of the adult human brain. Nat Neurosci 18, 1832-1844

41 Thompson, C.L. et al. (2014) A High-Resolution Spatiotemporal Atlas of Gene Expression of the Developing Mouse Brain. Neuron 83, 309-323

42 Parkes, L. et al. (2017) Transcriptional signatures of connectomic subregions of the human striatum. Genes Brain Behav 16, 647-663

43 Bernard, A. et al. (2012) Transcriptional architecture of the primate neocortex. Neuron 73, 1083-1099

44 (2012) Spatio-temporal transcriptome of the human brain. 478, 483-489

45 Lein, E.S. et al. (2017) Transcriptomic Perspectives on Neocortical Structure, Development, Evolution, and Disease. Annu Rev Neurosci 40, 629-652

46 Miller, J.A. et al. (2014) Transcriptional landscape of the prenatal human brain. Nature 508, 199-206

47 Zapala, M.A. et al. (2005) Adult mouse brain gene expression patterns bear an embryologic imprint. Proc Natl Acad Sci USA 102, 10357-10362

Kirsch, L. and Chechik, G. (2016) On Expression Patterns and Developmental Origin of Human Brain Regions. PLoS Comp Biol 12, e1005064-25

Cahalane, D.J. et al. (2012) Systematic, balancing gradients in neuron density and number across the primate isocortex. Front. Neuroanat. 6, 28

Charvet, C.J. et al. (2014) Systematic, Cross-Cortex Variation in Neuron Numbers in Rodents and Primates. Cereb Cortex 25, 147-160

51 Finlay, B.L. and Uchiyama, R. (2015) Developmental mechanisms channeling cortical evolution. Trends Neurosci 38, 69-76

52 MacLean, E.L. et al. (2014) The evolution of self-control. Proc Natl Acad Sci USA 111, E2140-E2148

53 Stevens, J.R. (2014) Evolutionary pressures on primate intertemporal choice. Proc. Biol. Sci. 281, 20140499-20140499

54 Cahalane, D.J. et al. (2014) Modeling local and cross-species neuron number variations in the cerebral cortex as arising from a common mechanism. Proc Natl Acad Sci USA 111, 17642-17647

55 Charvet, C.J. and Finlay, B.L. (2014) Evo-Devo and the Primate Isocortex: The Central Organizing Role of Intrinsic Gradients of Neurogenesis. Brain Behav Evol 84, 81-92 
Chen, C.-H. et al. (2011) Genetic Influences on Cortical Regionalization in the Human Brain. Neuron 72, 537-544

57 Badre, D. and D'Esposito, M. (2009) Is the rostro-caudal axis of the frontal lobe hierarchical? Nat Rev Neurosci 10, 659-669

58 Margulies, D.S. et al. (2016) Situating the default-mode network along a principal gradient of macroscale cortical organization. Proc Natl Acad Sci USA 113, 12574-12579

59 Huntenburg, J.M. et al. (2017) A Systematic Relationship Between Functional Connectivity and Intracortical Myelin in the Human Cerebral Cortex. Cereb Cortex 27, 981-997

60 Goulas, A. et al. (2018) Cortical Gradients and Laminar Projections in Mammals. Trends Neurosci DOI: 10.1016/j.tins.2018.06.003

61 Bastos, A.M. et al. (2012) Canonical Microcircuits for Predictive Coding. Neuron 76, 695-711

62 Markov, N.T. and Kennedy, H. (2013) The importance of being hierarchical. Curr Opin Neurobiol 23, 187194

63 Mesulam, M.M. (1998) From sensation to cognition. Brain 121, 1013-1052

64 Buckner, R.L. and Krienen, F.M. (2013) The evolution of distributed association networks in the human brain. Trends Cogn Sci 17, 648-665

65 Fulcher, B.D. et al. (2018) Multimodal gradients across mouse cortex. bioRxiv 10, 1-7

66 Burt, J.B. et al. (2018) Hierarchy of transcriptomic specialization across human cortex captured by structural neuroimaging topography. Nat Neurosci 27, 889-15

67 Maunsell, J.H. et al. (1983) The connections of the middle temporal visual area (MT) and their relationship to a cortical hierarchy in the macaque monkey. J Neurosci 3, 2563-2586

68 Felleman, D.J. and Van Essen, D.C. (1991) Distributed hierarchical processing in the primate cerebral cortex. Cereb Cortex 1, 1-47

69 Markov, N.T. et al. (2013) Anatomy of hierarchy: Feedforward and feedback pathways in macaque visual cortex. J Comp Neurol 522, 225-259

70 Belgard, T.G. et al. (2011) A Transcriptomic Atlas of Mouse Neocortical Layers. Neuron 71, 605-616

71 He, Z. et al. (2017) Comprehensive transcriptome analysis of neocortical layers in humans, chimpanzees and macaques. Nat Neurosci 20, 886-895

72 Marín-Padilla, M. (1992) Ontogenesis of the pyramidal cell of the mammalian neocortex and developmental cytoarchitectonics: a unifying theory. J Comp Neurol 321, 223-240

73 Zeng, H. et al. (2012) Large-scale cellular-resolution gene profiling in human neocortex reveals speciesspecific molecular signatures. Cell 149, 483-496

74 Reardon, P.K. et al. (2018) Normative brain size variation and brain shape diversity in humans. Science 360, 1222-1227

Glasser, M.F. and Van Essen, D.C. (2011) Mapping human cortical areas in vivo based on myelin content as revealed by T1- and T2-weighted MRI. J Neurosci 31, 11597-11616

Sperry, R. (1963) Chemoaffinity in the orderly growth of nerve fiber patterns and connections. Proc Natl Acad Sci USA

Dickson, B.J. (2002) Molecular mechanisms of axon guidance. Science 298, 1959-1964

$\mathrm{Ji}$, S. et al. (2014) Integrative analysis of the connectivity and gene expression atlases in the mouse brain. Neurolmage 84, 245-253

80 Fakhry, A. and Ji, S. (2015) High-resolution prediction of mouse brain connectivity using gene expression patterns. Methods 73, 71-78

81 Romero-Garcia, R. et al. (2018) Synaptic and transcriptionally downregulated genes are associated with cortical thickness differences in autism. Mol Psychiatry DOI: 10.1038/s41380-018-0023-7

82 Vértes, P.E. et al. (2016) Gene transcription profiles associated with inter-modular hubs and connection distance in human functional magnetic resonance imaging networks. Philosophical Transactions of the Royal Society B: Biological Sciences 371, 20150362-13

83 Seidlitz, J. et al. (2017) Morphometric Similarity Networks Detect Microscale Cortical Organization and Predict Inter- Individual Cognitive Variation. Neuron DOI: 10.1016/j.neuron.2017.11.039 
84 Varadan, V. et al. (2006) Computational inference of the molecular logic for synaptic connectivity in C. elegans. Bioinformatics 22, e497-e506

85 Baruch, L. et al. (2008) Using expression profiles of Caenorhabditis elegans neurons to identify genes that mediate synaptic connectivity. PLoS Comp Biol 4, e1000120

Kaufman, A. et al. (2006) Gene expression of Caenorhabditis elegans neurons carries information on their synaptic connectivity. PLoS Comp Biol 2, e167

Wolf, L. et al. (2011) Gene Expression in the Rodent Brain is Associated with Its Regional Connectivity. PLoS Comp Biol 7, e1002040-10

Mills, B.D. et al. (2018) Correlated Gene Expression and Anatomical Communication Support Synchronized Brain Activity in the Mouse Functional Connectome. J Neurosci 38, 5774-5787

Zalesky, A. and Fornito, A. (2009) A DTI-derived measure of cortico-cortical connectivity. IEEE Trans Med Imaging 28, 1023-1036

Zalesky, A. et al. (2012) On the use of correlation as a measure of network connectivity. Neurolmage 60, 2096-2106

91 Wang, G.-Z. et al. (2015) Correspondence between Resting-State Activity and Brain Gene Expression. Neuron 88, 659-666

92 Sethi, S.S. et al. (2017) Structural connectome topology relates to regional BOLD signal dynamics in the mouse brain. Chaos 27, 047405-15

93 Richiardi, J. et al. (2015) Correlated gene expression supports synchronous activity in brain networks. Science 348, 1241-1244

94 Pantazatos, S.P. and Li, X. (2017) Commentary: BRAIN NETWORKS. Correlated Gene Expression Supports Synchronous Activity in Brain Networks. Science 348, 1241-4. Front. Neurosci. 11, e1001049-4

95 Richiardi, J. et al. (2017) Distance Is Not Everything In Imaging Genomics Of Functional Networks: Reply To A Commentary On Correlated Gene Expression Supports Synchronous Activity In Brain Networks. bioRxiv DOI: $10.1101 / 132746$

96 Rubinov, M. et al. (2015) Wiring cost and topological participation of the mouse brain connectome. Proc Natl Acad Sci USA 112, 10032-10037

97 Mišić, B. et al. (2014) Communication efficiency and congestion of signal traffic in large-scale brain networks. PLoS Comp Biol 10, e1003427

98 Vaishnavi, S.N. et al. (2010) Regional aerobic glycolysis in the human brain. Proc Natl Acad Sci USA 107, 17757-17762

99 Liang, X. et al. (2013) Coupling of functional connectivity and regional cerebral blood flow reveals a physiological basis for network hubs of the human brain. Proc Natl Acad Sci USA 110, 1929-1934

100 Collin, G. et al. (2014) Structural and functional aspects relating to cost and benefit of rich club organization in the human cerebral cortex. Cereb Cortex 24, 2258-2267

101 van den Heuvel, M.P. and Sporns, O. (2013) Network hubs in the human brain. Trends Cogn Sci 17, 683696

102 Zamora-López, G. et al. (2010) Cortical hubs form a module for multisensory integration on top of the hierarchy of cortical networks. Front Neuroinform 4, 1

103 Fornito, A. et al. (2015) The connectomics of brain disorders. Nat Rev Neurosci 16, 159-172

104 Bullmore, E. et al. (2009) Generic aspects of complexity in brain imaging data and other biological systems. Neurolmage 47, 1125-1134

105 Bassett, D.S. et al. (2010) Efficient physical embedding of topologically complex information processing networks in brains and computer circuits. PLoS Comp Biol 6, e1000748

106 Meunier, D. et al. (2009) Hierarchical modularity in human brain functional networks. Front Neuroinform 3,37

107 Bassett, D.S. and Bullmore, E.T. (2016) Small-World Brain Networks Revisited. The Neuroscientist 23, 499-516

108 van den Heuvel, M.P. and Sporns, O. (2011) Rich-club organization of the human connectome. J Neurosci 31, 15775-15786 
109 van den Heuvel, M.P. et al. (2012) High-cost, high-capacity backbone for global brain communication. Proc Natl Acad Sci USA 109, 11372-11377

110 Towlson, E.K. et al. (2013) The Rich Club of the C. elegans Neuronal Connectome. J Neurosci 33, 63806387

111 Sporns, O. and Betzel, R.F. (2016) Modular Brain Networks. Annu. Rev. Psychol. 67, 613-640

112 Fornito, A. et al. (2013) Graph analysis of the human connectome: promise, progress, and pitfalls. Neurolmage 80, 426-444

113 Betzel, R.F. and Bassett, D.S. (2018) Specificity and robustness of long-distance connections in weighted, interareal connectomes. Proc Natl Acad Sci USA 115, E4880-E4889

114 Gal, E. et al. (2017) Rich cell-type-specific network topology in neocortical microcircuitry. Nat Neurosci 20, 1004-1013

115 Markov, N.T. et al. (2014) A weighted and directed interareal connectivity matrix for macaque cerebral cortex. Cereb Cortex 24, 17-36

116 Jaluria, P. et al. (2007) A perspective on microarrays: current applications, pitfalls, and potential uses. Microb. Cell Fact. 6, 4

117 Ziegenhain, C. et al. (2017) Comparative Analysis of Single-Cell RNA Sequencing Methods. Mol. Cell 65, 631-643.e4

118 Oldham, M.C. et al. (2008) Functional organization of the transcriptome in human brain. Nat Neurosci $11,1271-1282$

119 Zhu, Z. et al. (2016) Integration of summary data from GWAS and eQTL studies predicts complex trait gene targets. Nat Genet 48, 481-487

120 Goyal, M.S. et al. (2014) Aerobic Glycolysis in the Human Brain Is Associated with Development and Neotenous Gene Expression. Cell Metabolism 19, 49-57

121 Colantuoni, C. et al. (2012) Temporal dynamics and genetic control of transcription in the human prefrontal cortex. Nature 478, 519-523

122 Bakken, T.E. et al. (2016) A comprehensive transcriptional map of primate brain development. Nature 535, 367-375

123 Whitaker, K.J. et al. (2016) Adolescence is associated with genomically patterned consolidation of the hubs of the human brain connectome. Proc Natl Acad Sci USA 113, 9105-9110

124 Tasic, B. et al. (2016) Adult mouse cortical cell taxonomy revealed by single cell transcriptomics. Nat Neurosci 19, 335-346

125 McColgan, P. et al. (2018) Brain Regions Showing White Matter Loss in Huntington's Disease Are Enriched for Synaptic and Metabolic Genes. Biol Psychiatry 83, 456-465

126 Romme, I.A.C. et al. (2017) Connectome Disconnectivity and Cortical Gene Expression in Patients With Schizophrenia. Biol Psychiatry 81, 495-502

127 Rittman, T. et al. (2016) Regional expression of the MAPT gene is associated with loss of hubs in brain networks and cognitive impairment in Parkinson disease and progressive supranuclear palsy. Neurobiology of Aging 48, 153-160

128 Mclnnes, L. and Healy, J. UMAP: Uniform Manifold Approximation and Projection for Dimension Reduction., arXiv.org. (1802)

129 Oh, S.W. et al. (2014) A mesoscale connectome of the mouse brain. Nature 508, 207-214 